\title{
Rethinking heart failure care and health technologies from early COVID-19 experiences - A narrative review
}

\author{
Sakine Satici ${ }^{1}$, Pupalan lyngkaran ${ }^{2}$, Sharon Andrew ${ }^{1}$, Arun Patil $^{3}$, Niranjan Bidargaddi $^{4}$, Malcolm Battersby $^{5}$, \\ Maximilian De Courten ${ }^{1, *}$ \\ ${ }^{1}$ Victoria University, PO Box 14428 Melbourne Victoria 8001, Melbourne, Australia \\ ${ }^{2}$ University of Notre Dame, Werribee Mercy Sub School, School of Medicine Sydney, 300 Princes Hwy, Werribee VIC 3030 , Australia \\ ${ }^{3}$ Academic Dean, Victorian Institute of Technology, Level 14, 123 Queen Street, Melbourne, Victoria 3000, Australia \\ ${ }^{4}$ Flinders University \& SAHMRI, Sturt Rd, Bedford Park SA 5042, Australia \\ ${ }^{5}$ Professor and Head of Psychiatry, College of Medicine and Public Health, Flinders University, Flinders Medical Centre, Fli nders Drive, Bedford Park SA 5042 \\ GPO Box 2100, Adelaide SA 5001, Australia \\ *Correspondence: maximilian.decourten@vu.edu.au (Maximilian De Courten)
}

DOI:10.31083/j.rcm.2021.01.272

This is an open access article under the CC BY 4.0 license (https://creativecommons.org/licenses/by/4.0/).

Submitted: 04 December 2020 Revised: 04 March 2021 Accepted: 04 March 2021 Published: 30 March 2021

Heart Failure (HF), a common chronic disease, requires multidisciplinary care to optimise outcomes. The COVID-19 pandemic, its impact on people's movement and access to health services, introduced severe challenges to chronic disease management. The era that will evolve after this pandemic is likely to provide uncertainty and service model disruptions. HF treatment is based on guidelines derived from randomised clinical trial evidence. Translational shortfalls from trials into practice have been overcome with post-trial service improvement studies like OPTIMIZE-HF where a team using a process of care can translate evidence to the general population. However, gaps remain for vulnerable populations e.g. those with more severe HF, with multiple comorbid conditions, and certain demographic groups and/or residents in remote locations. Health technology has come with great promise, to fill some of these gaps. The COVID-19 pandemic provides an opportunity to observe, from Australian healthcare lens, $\mathrm{HF}$ management outside the traditional model of care. This narrative review describes relatively recent events with health technology as a solution to improve on service gaps.

Keywords

Congestive heart failure; Chronic disease management; Heart failure; Selfmanagement; Health hubs; Health technology

\section{Introduction}

A disruptive force, COVID-19 affecting 185 countries and $>3$ million patients (April 2020), will highlight resilience and expose deficiencies in health systems. It rivals the Spanish flu of 1918 as a modern public health epidemic. Predominately respiratory based with pneumonia and respiratory failure, multiorgan involvement including cardiovascular are observed. Heart failure (HF), myocardial injury and arrhythmias are most common cardiac complications; in 339 critical care elderly, $17.4 \%$ suffered HF [1-4]. At a population level the effects was sudden, prolonged, where little was known and finally aggravated by limititations to movement and reduced access to health care $[5,6]$. From the authors focus on heart failure (HF) care in Australia, we believe:

"The most important goal in this area is summarised in the term 'ambulatory' and delivering a working space at the provider and recipient ends to monitor, diagnose and deliver safe management that complements and reduces cost of entirely centre based care."

When dealing with uncertainty outside a hospital environment, a process of care is important as the foundation, structure and pathway to guide the many elements required to deliver successful chronic disease care. The specifics of HF management are summarised in consensus guidelines $[7,8]$ and disease management [9], This backbone of disease management complements guideline based recommendations. The HF team must then juggle these resources to provide optimal service for individual clients. COVID-19 has brought some issues to the fore:

1. CHF patients are heterogenous, hence single option models may not deliver uniform outcomes. Where more options are available training and resourcing adds to higher costs;

2. The circumstances for some patients and health services may not be compatible with hosting all management options;

3. In prioritising health resources there will be groups whose services are disproportionately lower compared to their needs [10].

Nonetheless, this universal event provides a unique opportunity for observations and reflection of HF management and health technologies 'ambulatory home health hubs'. These challenges should provide optimism that what we can learn lesson to close existing gaps and improve care [11]. In this narrative review we explore the chronic HF model including self-management capacities and health technology exposed by COVID-19 pandemic. 


\section{Chronic care concepts, evidence and translation for congestive heart failure}

Barr et al. in 2003 presented an expanded chronic care model (CCM) arguing that the language of generic CCM was not resonating with health practitioners $[12,13]$. The authors argue in this paper that the term 'resonate', a largely qualitative term, has an influence on every CCM every domain. Fig. $1^{1}$, we have broken down the core pillars in this model. Disease management domains have numerous subdomains that deal with complicated and qualitative areas. When these issues are not resolved adequately, the problem can grow exponentially. Similarly, domains with many subdomains or variables can add stress to service delivery.

In Table $1^{1}$, when we consider integrating health technologies into a heart failure CCM, we must factor this gap. In reality, health innovation involving technology and chronic disease self-management programs (CDSMP), have limited evidence and suffer poor translation. In Australia at the height of the pandemic, a payment code was created to facilitate phone consultation with patients, where evidence is limited. Thus, it will be interesting to observe uncontrolled care models implemented during this pandemic. there are also important limitations that need to be factored when using the CCM [14-19].

a. CCM preconditions for patient's willingness and abilities and health care team capabilities to respond, positively e.g. as demonstrated in diabetic care [16].

b. CCM Flexibility - guideline-directed chronic disease care does not appear consistently in real world disease management programs [17].

c. CCM Integration - cost-effectiveness is better served if strategies for disease management, prevention and promotion utilise similar design principles $[9,17]$.

d. CCM collaboration or choice - between policy, community resources and broad patient-health system 'resonance' may need to be expanded to account with day-to-day scenarios [17].

e. CCM Links - is required to ensure dialogue with communities in developing strategies particularly health prevention and promotion.

f. CCM Outcomes - clear outcomes must be determined for the program e.g. cost saving and reducing readmissions are important and occasionally under played goal particularly in large trial settings.

In this table we assume a scenario of client, case manager and hospital. All interactions use some form of technology (i.e. no postal or mail). There are seven clinical scenarios. The resourcing model highlights the most likely resources to

1 The points raised in Fig. 1 and Table 1 are established and previously published. However, the focus of each figure represents the stage of thinking in the process of implementing a CCM for heart failure and health technologies There may be overlap between some points in the different figures as they are presented unchanged from the references. be aid that scenario. Resources are Health technologies, Personnel, and health information technology.

- Telemedicine (Structured Telephone Support-STS) is defined as the direct provision of clinical care, including diagnosing, treating, or consultation, via telecommunications for a patient at a distance. Its primary function is to provide specialist consultation to distant communities, rather than to provide a tool for self-management of chronic disease [22].

- Telecare (Telemonitoring) focused on providing care in a home setting with the primary intent of supporting the patient rather than the health professionals [22].

- Home telemonitoring (TM) (remote monitoring $\mathbf{R M}$ ) is used in a more restrictive sense and encompasses the use of audio, video, and other telecommunication technologies to monitor patient status at a distance.

Participants, technology domains, evidence base and translational strategy links are highlighted. For effective models, the right balance between health systems support, patient self-management capabilities and flexible research and implementation strategies must exist. Balancing quantitative and qualitative research is also necessary to deliver broad data.

As medicine will experience many changes in the coming years, this "medicine of the future" will be increasingly proactive, featuring four basic elements: predictive, personalized, preventive, and participatory.

\section{Heart failure chronic care model under prolonged forced isolation}

The clinical environment in Melbourne, Australia, for a period saw only essential clinical services operating and a fear factor in utilising health services. All health services domains experienced challenges. When using technology, there are some similarities with medical practice early last century where individual practitioners dispense care from observation, or experience, as evidence was limited. Evidenced based medicine (EBM) also created silos in some respects [2022]. For e.g. the Australian Federal Government in response to self-isolation approved funding for telephone-based consulting, bypassing all the established processes highlighted in Fig. 1. This compares to conventional telemedicine for geographical distance, which is video conferenced, standardised by a list of protocols including patient side supports and haemodynamics. We must thus also explore technology with primary aim to enhance self-management capacities of patients and as an adjunct to breaking silo's, its secondary goal improve outcomes, while maintaining an acceptable standard of EBM.

\subsection{COVID-19 and heart failure}

The mechanism of virus pathophysiology are addressed in these papers $[1,2,5,23]$. The exact COVID-19 cardiovascular interplay remains unclear, with $\mathrm{CV}$ complications reported in many elderly and especially with pre-existing comorbidities. Three pooled reviews with COVID-19 infected patients show: Kunetsor et al., studied 5815 patients in 17 retrospective cohort studies. The average age ranged from 


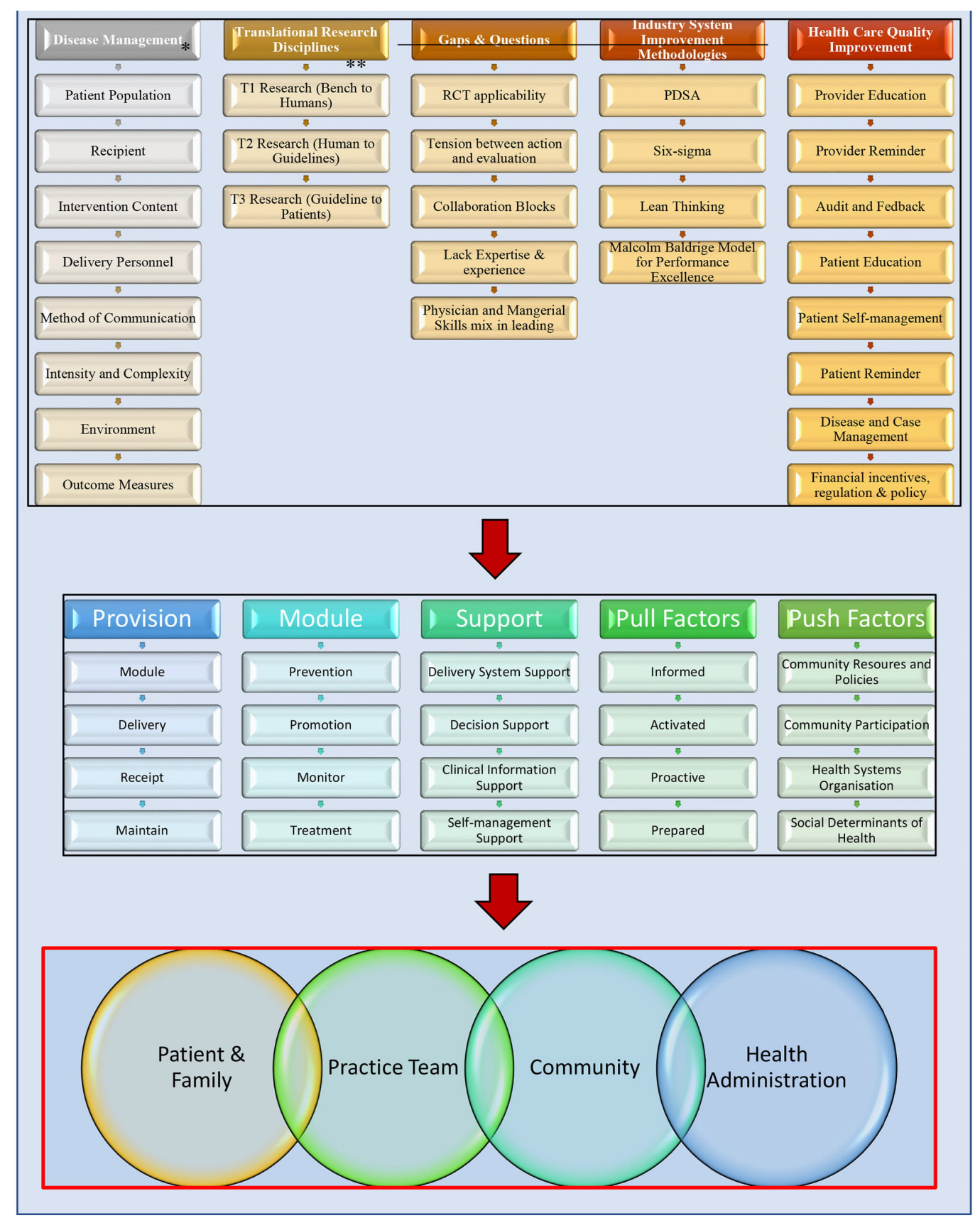

Fig. 1. Components of Disease Management and Steps in Translating and Implementing Heart Failure Models. The chronic care model has many domains and subdomains. Translating evidence is at the heart of evidenced based medical care. Three phases: firstly, process and quality of care standards; secondly creating models on regional needs; and finally, health policy and translation to stakeholders. Some areas are more subjective than others and finding evidence to satisfy the process can be difficult. ${ }^{*}$ Disease Management: 1. Patient Population - Risk Status, Comorbid Condition, Non-clinical features; 2. Recipient - Patient/Caregiver; Case Provider; 3. Intervention Content - Patient/Caregiver Education, Medical Management, Peer Support, Remote Monitoring; 4. Delivery Personnel - Nurse, Physician, Pharmacist, social workers, Dieticians Physical therapists, Psychologists, Case managers, Care coordinators; 5. Method of Communication - Face-to-face individual, Face-to-face group, Telephone in person, Telephone Mechanised, Internet; 6. Intensity and Complexity - Duration, Frequency/Periodicity, Complexity; 7. Environment - Hospital in-patient, Hospital out-patient, Home-based; 8. Outcome Measures - Clinical measures, Process Measures, Quality of life measures, Patient Satisfaction, Provider Satisfaction. ${ }^{* *}$ Translational Research Disciplines: 1 . T1 Bench to Human - Basic Science, Molecular Biology, Genetics, Technology Assessment, Animal Research, Phase I \& II clinical trials; 2. T2 Human to Guideline Phase 3 clinical trials, observational studies, evidence synthesis and guidelines, clinical epidemiology, comparative effectiveness, policy and ethics; 3 . T3 Guideline to Patient - Implementation and Dissemination, systems redesign, communication theory, behavioural and management science, organizational development, patient encounter research (concepts modified from ref [7-16]). 
47 to 71 years. The prevalence of pre-existing CVD was 14.6\% (11.0-18.4; 95\% CI 87, 94\%; $P<0.01)$; HF overstays $17.6 \%(14.2-21.2$; $95 \%$ CI $0,76 \%$; $P=0.20)$ for HF; myocardial injury, had worse outcomes in older age and with preexisting comorbidities [2]; Sabatino et al. pooled 21 studies with 77317 hospitalized patients, $12.86 \%$ had CV comorbidities; $14.09 \%$ developed CV complications during hospitalization. Pre-existing cardiovascular comorbidities were significantly associated to $\mathrm{CV}$ complications $(P=0.019)$; death $(P$ $=0.038)$ was associated with pre-existing cardiovascular comorbidities $(P<0.001)$, older age $(P<0.001)$, and the development of cardiovascular complications during the hospitalization [4]. Bae et al. pooled 51 studies with 48,317 infections where death or severe illness was significantly higher in patients with risk factors for CVD (hypertension: OR 2.50, 95\% CI 2.15 to 2.90; diabetes: 2.25 , 95\% CI 1.89 to 2.69$)$ and CVD (3.11, 95\% CI 2.55 to 3.79$)$ [24].

HF patients also experience greater barriers in service access, including the fear of utilising a spectrum of services such as rehabilitation, pharmacotherapies and devices. Novel observations on pathophysiology e.g. on ACE2. This enzyme is highly expressed in the heart, and states that cause excessive activation of the renin-angiotensin system, such as hypertension, $\mathrm{HF}$, and atherosclerosis $[1,5,23]$, may counteract the effects of angiotensin II. We anticipate prospective studies to add to this space.

\subsection{The heart failure team as a cardiologist led chronic disease service}

The HF team shares features with all chronic disease teams i.e. allied health, primary care and other specialty input. The cardiologist are appropriate leads as CHF pathosphysiology is complex. Specifically, both forms of HF, reduced (HFrEF) or preserved (HFpEF) cardiac function share similar prevalence's, but older age and female sex predominates with HFpEF. Multimorbidity e.g. coronary artery disease, diabetes mellitus, chronic renal impairment, hypertension, are seen in more than half of patients. Patient socioeconomics, demography, geography, and distribution of health services add to the management complexity [19]. Hence a cardiology lead with a good link and dynamic link to other providers is important. Many of the HF program goals can be addressed when patients live within proximity to hospital services; in fact, many other areas of the programs are redundant for achieving optimal care; however as patients gravitate further (either geographical, personal preference, social isolation, etc.) more services will be absorbed by primary health, community specialist, community hospitals, hospital outreach and even by 'good self-managing' patients. However the gold standard is hospital-based model of care, that works well for all patient types and the evidenced tested is also weighted toward this model [16].

The HF Program while utilising the CCM concepts has differences in how its implemented compared to other chronic ailments. Fundamentally the HF team sees the cardiologist addressing disease specific issues; with assistance from a team that includes a nurse, allied health, primary and other medical specialist care on $\mathrm{HF}$ and chronic disease issues $[7,8,11]$. While models have explored primary care and allied health leads, one clear observation from COVID-19 is that in reality, the HF team is a 'cardiologist led' chronic disease team as the latter steers more prognostic issues related to HF. While consolidation of key management areas requires the cardiologist, the sharing of broader care with GP, allied health, is significant for completing the care with planning, information sharing, delivering aspects of care or even handling unforeseen situations. Should patients have comorbidities, differentiation of who leads the team is best mutually agreed based on the primary disease with the greatest burden on quality of life, morbidity, and mortality. In institutions, in-hospital cardiologists or HF subspecialist leads the team. However, in community practice this delineation is less clear. The fundamental difference is access to allied and sub-speciality services. The hospital-based HF specialist can access, refer, prescribe most care, but in the community the general practitioner has greater access to these services. This is an important point as the model of care also various significantly with the type of chronic disease even though many overlapping features are common. There are thus often multiple care models however funding models more likely favour the hospital led concepts.

\subsection{Self-management in heart failure}

'Self-management' introduced in the 1960's in paediatric chronic asthma sufferers, has successes in pain management, diabetes and hypertension. The terminology is widely applied in CDSMP as a core component to engaging patients in being actively involved in the day-to-day management of their chronic condition. However, despite significant attention to promoting self-management there has been poor translational success into patients with HF being actively engaged and good self-managers, despite generic HF-CDMP having selective successes. CDSMP is a problem based, approach designed to achieve disease: i) maintenance; ii) management; and iii) efficacy. Such programs create the structures to encourage patient's engagement with the day-to-day and long-term management of their chronic illness. Selfmanagement successes are measured by the levels of selfefficacy and self-tailoring achieved. CDSMP comprise of three key components [25]:

1. Goals - comprising of: i) performance mastery; ii) modelling; iii) interpretation of symptoms; and iv) social persuasion.

2. Tasks - comprising of: i) medical management; ii) role management; and iii) emotional management.

3. Skills - comprising of: i) problem solving; ii) decision making; iii) resource utilization; iv) forming a patient/health care provider partnership; and v) taking action.

Anecdotally, self-management capacities of self-isolating patients would have increased, unfortunately there are no data on its impact on worsening HF. None the less in all the studies and trials to date, for HF, while there have been 
positive findings, no health system to date has successfully translated a viable model of CDSMP that impacts on major adverse events, outcomes or cost effectiveness [25-29]. The foundations of managing readmissions in health services around self-management is also proving difficult largely because there is no standardised model that traverses the boundaries of patients' journeys. CDSMP however must link to health technologies for effective models, and there will be lessons to learn from patients' experiences.

\section{Ambulatory health technologies in congestive heart failure \\ 4.1 Established technologies}

Technology assisted communications in stable situations have been established for decades using telephone, mobile messaging, or emails. Greater scope with video conferencing technologies has also allowed remote teleconsultations. In Australia, during the COVID-19 pandemic and social isolation, Medicare created a funded solution for teleconsultations with patients disregarding previous geographical limitations and video conferencing [30]. This unplanned experiment provides opportunity to study this concept for social isolation and special circumstances to balance mobility and even work commitments in the overall health economic equation. What was however missing between routine teleconsultation and the present was a protocol. There were no standards for consultations and no ability to obtain examination findings to equate to a face-to-face review. Inglis et al. followed up a 2010 Cochrane publication reviewing randomised controlled trials (RCTs) of structured telephone support or non-invasive home telemonitoring compared to standard practice for patients with CHF, to evaluate the cumulative efficacy of intervention and usual care. Twenty-five studies with 9332 participants for structured telephone support reduced mortality, HF hospitalisations, quality of life, knowledge of HF and selfcare capacity. Age did not alter capacity and ease in learning technology or satisfaction. The quality of evidence did however vary from low to moderate [31]. Other established technologies include centre-based diagnostics, routine clinicbased tools and established ambulatory implanted treatment devices [32], are not a focus of this review.

\subsection{Technology in trials and translation}

Ambulatory technology for advanced communications including telemonitoring, diagnostics and assisting non-device (programmed) therapies has seen a large number of studies, protocols, and outcomes explored and with mixed results reported [33-35]. Outside trial settings most patients with HF will have non standardised devices to measure or monitor heart rates, blood pressure and weight. Implanted devices have capacity to assess lung parenchymal fluid overload and in conjunction with right heart catheters have been trialled for early detection of rising pulmonary capillary wedge pressures. Pare et al. reviewed 65 empirical studies on cardiac and comorbid conditions found that regardless of nationality, socioeconomic status, or age, patients comply with telemonitoring programs the use of technologies for ambulatory care. At this stage in 2007 there were variations in favour of cardio-respiratory conditions without clear supportive cost-benefit evidence. The authors were able to conclude the technologies facilitated accurate and reliable data, empowers, and influences patient attitudes and behaviours, and may even improve health outcomes [36, 37]. Since the early days large costly devices collecting very limited homebased data, stored within cloud systems, remotely accessed by health team has been replaced by sophisticated integrated systems with a range of automated, algorithm or human activation, processing or problem solving has evolved. These led to the foundations of the newer large randomised controlled trials (RCT). These trials then also opened newer and larger issues with technologies included protocol, standards and training, data support, storage, ownership, platform interphase and population level implementation. The call for generic and user-friendly systems is ongoing [38].

Telemonitoring for treatments requires a greater understanding of terminology and medical physiology and experience to action findings. Telemedicine and remote monitoring represent more than the remote system-patient communication of health data, several reviews explore this in greater detail $[33,39,40]$. Essentially the ability to provide prevention, treatment and promotion requires a sophistication and accuracy in technology, and simplicity to increase uptake, before even exploring hard health outcomes measures like readmissions, morbidity, and mortality. With this frame of thinking we can then understand the variable outcomes seen in larger randomised trials ( $>250$ patients) the CHAT, TIM-HF, TEN-HMS and BEAT-HF, Tele-HF, INH, WISH, TEHAF studies [41-58]. Interpreting the success are interesting when we explore this early study by Gattis et al., by introducing a clinical pharmacist into the heart failure team improves CHF outcomes [58]. Clearly defining the HF team as multidisciplinary we can add positive assets that lead to outcomes. However, among the non-invasive studies only TIM-HF2, with 1571 participants and the most comprehensive parameters measured, web-based remote monitoring on daily weight, BP, pulse, ECG, peripheral capillary oxygen saturation, a self-related health status, showed reduction in $\mathrm{HF}$ admissions or death (HR 0.80; 95\% CI 0.65-1.00). All the other trials had patients with different characteristics, different protocols, tools, endpoints and mechanism of followup making it difficult to standardise the findings. However, when we break down performance measures daily weight and voice calls have high value $[44,45]$.

The most novel are mobile phone and devices. Cajita et al. looked at 10 articles utilized mobile health technology as part of a HF monitoring system, including blood pressure measuring device, weighing scale, and an ECG recorder found inconsistent outcomes between studies from sample-size, study design, older technologies [44, 58]. Inglis et al. has submitted a Cochrane protocol and we await that finding [31]. Implanted Device platforms and invasive monitoring as trialled 
Table 1. Executing CCM using technology for HF.

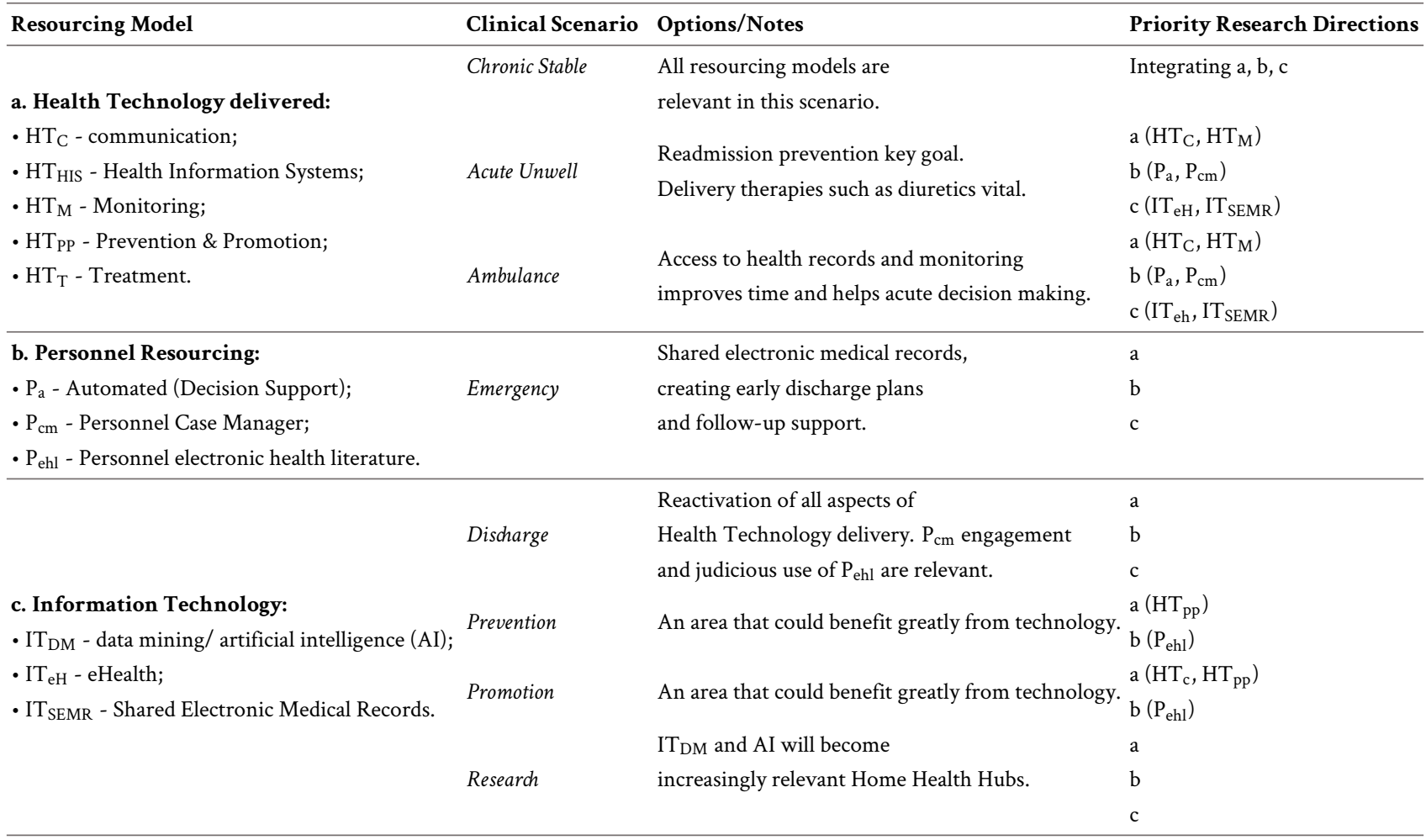

in DOT-HF, REM-HF, MORE-CARE, OptiLink HF, EFFECT, IN-TIME, MultiSENSE, COMPASS-HF, LAPTOPHF, CHAMPION is an evolving field. The risks of infection and chronically implanted foreign objects add to the noninvasive translation issues. The results have been mixed, however it worth mentioning as it highlights increasing the remote data to equate high level hospital care alone is not sufficient to reproduce institutional outcomes in the ambulatory setting [45]. Health promotion and prevention stems from secondary prevention clinics. This is usually a part of cardiac rehabilitation, general practice, and specialist consultation. Dedicating time for this is part of the heart failure program. However, to achieve parity with other chronic diseases, motivational interviewing, and cognitive behavioural therapies to improve quality of life and change negative behaviours is required. In Australia very limited programs are capable of offering this and outside public systems such models are not remunerated.

Kacrowski et al. randomised 39 communities to Cardiovascular Health Awareness Program (CHAP), using a limited number of ambulatory technologies. The CHAP communities, volunteer run (adults $>65$ years) cardiovascular risk assessment and education sessions in community pharmacies over 10 weeks; automated blood pressure, self-reported risk factor were collected and shared with participants, physicians and pharmacists. This collaborative, community based health promotion and prevention programme reduce cardiovascular morbidity and hospital admission (including CHF) with a $9 \%$ relative reduction in composite end point (rate ratio
$0.91,95 \%$ confidence interval 0.86 to $0.97 ; P=0.002$ ) or 3.02 fewer annual hospital admissions for cardiovascular disease per 1000 people aged 65 and over [59]. In the TERVA study at 12 months of individualised health telephone coaching intervention in HF and other chronic diseases, 1221 participants, did not deliver the majority of disease management clinical measures. The authors concluded to provide substantial benefits, interventions may need to be more intensive, target specific sub-groups, and/or to be fully integrated into local health care [60]. In another home-based telephone coaching study among the $660 \mathrm{HF}$ participants mortality between IG and CG campaign was $(\mathrm{OR}=0.44 ; P=0.001)$ [61]. Titration of HF medication that is nurse lead achieves target dose in shorter intervals and improves readmission and mortality outcomes [62]. In nurse-run, cardiologist or nurse practitioner-supervised clinic, HF medication via telephone can be achieved in up to $97 \%$ with improvement in left ventricular ejection fraction [63]. Automated telemedicine systems using algorithms have been successful in home up titration of beta-blockers.

\subsection{The necessity of health innovation and integration}

Health innovation is a broad term, often used to imply technology. For health innovation 'a hub' or a 'Precinct' is vital as a drawcard, whether it be for geographical, social or other forms of isolation requiring to be connected through other means (Fig. 2). Adding machine learning and data management is vital, but beyond the scope of this review [64]. Technology works in daily life; it is working during these crises; thus, the question is why not in health systems? 


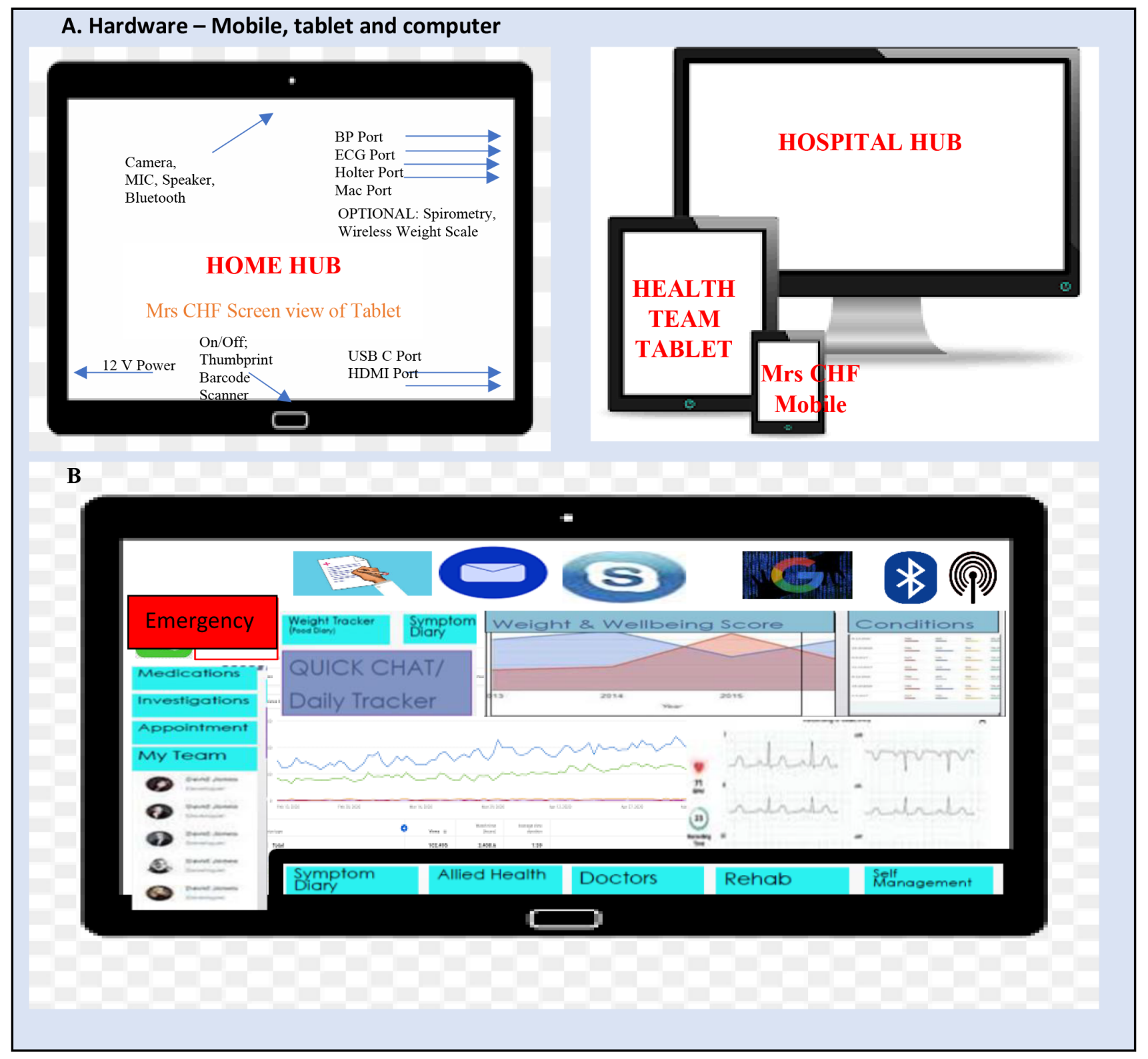

Fig. 2. Concepts for Integrated Health Hub. Both images are a theoretical concept of an Integrated Technology solution or 'Home Health Hub'. As highlighted, there are many domains for which technology can be used. We also discussed the need to reduce the number of variables in subdomains. (A) In this concept the hardware (above image) are limited to a home tablet. This is modified to house features for communication, monitoring and treatment. For portability, a mobile phone platform also interphases with the home system. The final interphase is located with the health care team; (B) This image shows the patients heath page. A range of information is displayed. A few actions are possible. This 'app' will be the command centre for patients and health care team. Again, it is important to minimise the variables while ensuring there is sufficient sophistication for the client's health needs. Alternatively, several versions tailored for greater self-management capabilities could be considerations.

There is an invisible wall regards social and political will to address medicolegal consequences and governance needed to push the boundaries. In CHF and CDSMP health innovation is needed in two key areas: firstly, finding a suitable model (like the hospital model) to administer, fund and remunerate, and then to risk stratify and tailor CDSMP's particularly on patients abilities from poor to good self-managers; secondly to find integrated technological platform as a hub to house patients side information in a user friendly manner and health system side in its natural complexity. This ca- pacity requires data storage, patient monitoring and communication. This set-up must factor the patient's illness journey. In HF there are stable periods, chronic deterioration or acute decompensation. The CDSMP and technology supported model must support clinical deterioration to keep patient's ambulatory and at home [36, 37]. Readmission reduction must be seen as a priority. Research framework must portray the real world picture of the question, and maintain stringent research methodology, and subsequent translation by health services $[16,17]$. Finally, health Integration (Fig. 1) 
of health innovation (Fig. 2) will be difficult area. We highlight 5 points for consideration.

1. Resonate - ie fit-for-purpose for clients and health services.

2. Understand - new terminologies and protocols.

3. Integrate - e.g. end bulky heavy systems are difficult to use.

4. Informed and activated - prepared, proactive clients and healthcare teams.

5. Standardise Program \& Performance Measure - know what to deliver and measure.

\section{Summary}

The COVID-19 has impacted health services and its significant impact on CHF care are coming to light. CHF being a chronic disease sees an acute hospital and stable community phases. On the ambulatory aspects traditional models of care built around face-to-face interactions, multidisciplinary input and multimodality treatments, makes this aspect quite complex. Aging, isolated, associated comorbidities and further challenges to traditional service models. The COVID-19 environment including self-isolation and access to services has tested self-management capacities and health technologies. However, from observations, paradigms could emerge. For e.g. if only to observe the technology uptake in all aspects of lives, it is clear, technology is here to stay. In medicine we see technology daily in the workplace. What has been most difficult is the ambulatory (home) and clinical workplace connect. We have seen patches of success. Very clearly technology will player a greater role. It is this important to put a structure in lay terms on approaching this. Undoubtedly this link between improved patient self-management, technology assistance and framing the right models around COVID-19 experiences, could add to improved chronic disease management.

\section{Author contributions}

SS and PI wrote the manuscript. All authors reviewed and provided feedback. MdC provided final oversight and approval.

\section{Acknowledgment}

The authors declare no acknowledgments.

\section{Funding}

This research received no external funding.

\section{Conflict of interest}

None pose a conflict of interest for this review.

\section{References}

[1] Akhmerov A, Marbán E. COVID-19 and the Heart. Circulation Research. 2020; 126: 1443-1455.

[2] Kunutsor SK, Laukkanen JA. Cardiovascular complications in COVID-19: a systematic review and meta-analysis. Journal of Infection. 2020; 81: e139-e141.
[3] Zhou F, Yu T, Du R, Fan G, Liu Y, Liu Z, et al. Clinical course and risk factors for mortality of adult inpatients with COVID-19 in Wuhan, China: a retrospective cohort study. Lancet. 2020; 395: 1054-1062.

[4] Sabatino J, De Rosa S, Di Salvo G, Indolfi C. Impact of cardiovascular risk profile on COVID-19 outcome. A meta-analysis. PLoS ONE. 2020; 15: e0237131.

[5] Clerkin KJ, Fried JA, Raikhelkar J, Sayer G, Griffin JM, Masoumi A, et al. COVID-19 and cardiovascular disease. Circulation. 2020; 141: $1648-1655$.

[6] Zaman S, MacIsaac AI, Jennings GL, Schlaich MP, Inglis SC, Arnold R, et al. Cardiovascular disease and COVID-19: Australian and New Zealand consensus statement. Medical Journal of Australia. 2020; 213: 182-187.

[7] Yancy CW, Jessup M, Bozkurt B, Butler J, Casey DE, Jr., Colvin $\mathrm{MM}$, et al. 2017 ACC/AHA/HFSA focused update of the 2013 ACCF/AHA guideline for the management of heart failure: a report of the American College of Cardiology/American Heart Association task force on clinical practice guidelines and the Heart Failure Society of America. Circulation. 2017; 136: e137-e161.

[8] Ponikowski P, Voors AA, Anker SD, Bueno H, Cleland JGF, Coats AJS, et al. 2016 ESC Guidelines for the diagnosis and treatment of acute and chronic heart failure: The Task Force for the diagnosis and treatment of acute and chronic heart failure of the European Society of Cardiology (ESC). Developed with the special contribution of the Heart Failure Association (HFA) of the ESC. European Heart Journal. 2016; 37: 2129-2200.

[9] Krumholz HM, Currie PM, Riegel B, Phillips CO, Peterson ED, Smith R, et al. A taxonomy for disease management: a scientific statement from the American Heart Association Disease Management Taxonomy Writing Group. Circulation. 2006; 114: 14321445.

[10] Iyngkaran P, Liew D, Neil C, Driscoll A, Marwick TH, Hare DL. Moving from heart failure guidelines to clinical practice: gaps contributing to readmissions in patients with multiple comorbidities and older age. Clinical Medicine Insights: Cardiology. 2018; 12 : 1179546818809358.

[11] Fonarow GC, Abraham WT, Albert NM, Stough WG, Gheorghiade M, Greenberg $\mathrm{BH}$, et al. Influence of a performanceimprovement initiative on quality of care for patients hospitalized with heart failure: results of the Organized Program to Initiate Lifesaving Treatment in Hospitalized Patients with Heart Failure (OPTIMIZE-HF). Archives of Internal Medicine. 2007; 167: 1493-1502.

[12] Barr V, Robinson S, Marin-Link B, Underhill L, Dotts A, Ravensdale $\mathrm{D}$, et al. The expanded chronic care model: an integration of concepts and strategies from population health promotion and the chronic care model. Healthcare Quarterly. 2003; 7: 73-82.

[13] Wagner EH, Glasgow RE, Davis C, Bonomi AE, Provost L, McCulloch D, et al. Quality improvement in chronic illness care: a collaborative approach. Joint Commission Journal on Quality Improvement. 2001; 27: 63-80.

[14] Arnett DK, Goodman RA, Halperin JL, Anderson JL, Parekh AK, Zoghbi WA. AHA/ACC/HHS strategies to enhance application of clinical practice guidelines in patients with cardiovascular disease and comorbid conditions: from the American Heart Association, American College of Cardiology, and US Department of Health and Human Services. Circulation. 2014; 130: 1662-1667.

[15] McCulloch DK, Price MJ, Hindmarsh M, Wagner EH. A population-based approach to diabetes management in a primary care setting: early results and lessons learned. Effective Clinical Practice. 1999; 1: 12-22.

[16] Wagner EH, Davis C, Schaefer J, Von Korff M, Austin B. A survey of leading chronic disease management programs: are they consistent with the literature? Managed Care Quarterly. 1999; 7: 56-66.

[17] Glasgow RE, Orleans CT, Wagner EH. Does the chronic care model serve also as a template for improving prevention? Milbank Quarterly. 2001; 79: 579-612. 
[18] Iyngkaran P, Majoni W, Cass A, Sanders P, Ronco C, Brady S, et al. Northern Territory perspectives on heart failure with comorbidities - understanding trial validity and exploring collaborative opportunities to broaden the evidence base. Heart, Lung \& Circulation. 2015 ; 24 : 536-543.

[19] Iyngkaran P, Harris M, Ilton M, Kangaharan N, Battersby M, Stewart $\mathrm{S}$, et al. Implementing guideline based heart failure care in the Northern Territory: challenges and solutions. Heart, Lung \& Circulation. 2014; 23: 391-406.

[20] Sheridan DJ, Julian DG. Achievements and limitations of evidence-based medicine. Journal of the American College of Cardiology. 2016; 68: 204-213.

[21] Evidence-Based Medicine Working Group. Evidence-based medicine: a new approach to teaching the practice of medicine. Journal of the American Medical Association. 1992; 268: 2420-2425.

[22] Loughlin M, Bluhm R, Buetow S, Upshur REG, Goldenberg MJ, Borgerson $\mathrm{K}$, et al. Reason and value: making reasoning fit for practice. Journal of Evaluation in Clinical Practice. 2012; 18: 929937.

[23] Nishiga M, Wang DW, Han Y, Lewis DB, Wu JC. COVID-19 and cardiovascular disease: from basic mechanisms to clinical perspectives. Nature Reviews Cardiology. 2020; 17: 543-558.

[24] Bae S, Kim SR, Kim M, Shim WJ, Park S. Impact of cardiovascular disease and risk factors on fatal outcomes in patients with COVID19 according to age: a systematic review and meta-analysis. Heart. 2021; 107: 373-380.

[25] Iyngkaran P, R. Toukhsati S, Harris M, Connors C, Kangaharan $\mathrm{N}$, Ilton $\mathrm{M}$, et al. Self managing heart failure in remote Australia translating concepts into clinical practice. Current Cardiology Reviews. 2016; 12: 270-284.

[26] Iyngkaran P, Majoni V, Nadarajan K, Haste M, Battersby M, Ilton $\mathrm{M}$, et al. AUStralian Indigenous Chronic Disease Optimisation Study (AUSI-CDS) prospective observational cohort study to determine if an established chronic disease health care model can be used to deliver better heart failure care among remote Indigenous Australians: Proof of concept-study rationale and protocol. Heart, Lung \& Circulation. 2013; 22: 930-939.

[27] Clark AM, Spaling M, Harkness K, Spiers J, Strachan PH, Thompson DR, et al. Determinants of effective heart failure self-care: a systematic review of patients' and caregivers' perceptions. Heart. 2014; 100: 716-721.

[28] Jonkman NH, Westland H, Groenwold RHH, Ågren S, Atienza F, Blue L, et al. Do Self-management interventions work in patients with heart failure? An individual patient data meta-analysis. Circulation. 2016; 133: 1189-1198.

[29] Toback M, Clark N. Strategies to improve self-management in heart failure patients. Contemporary Nurse. 2017; 53: 105-120.

[30] Australian Government. COVID-19 Temporary MBS Telehealth Services. 2020. Avaliable at: http://www.mbsonline.gov.au/inter net/mbsonline/publishing.nsf/Content/Factsheet-TempBB (Accessed: 01 October 2020).

[31] Inglis SC, Clark RA, Dierckx R, Prieto-Merino D, Cleland JGF. Structured telephone support or non-invasive telemonitoring for patients with heart failure. Cochrane Database of Systematic Reviews. 2015; 2015: CD007228.

[32] Hussein AA, Wilkoff BL. Cardiac implantable electronic device therapy in heart failure. Circulation Research. 2019; 124: 15841597.

[33] Sana F, Isselbacher EM, Singh JP, Heist EK, Pathik B, Armoundas AA. Wearable devices for ambulatory cardiac monitoring: JACC State-of-the-Art Review. Journal of the American College of Cardiology. 2020; 75: 1582-1592.

[34] Iyngkaran P, Toukhsati SR, Biddagardi N, Zimmet H, J Atherton J, Hare DL. Technology-assisted congestive heart failure care. Current Heart Failure Reports. 2015; 12: 173-186.

[35] Gonzalez Garcia M, Fatehi F, Bashi N, Varnfield M, Iyngkaran P,
Driscoll A, et al. A review of randomized controlled trials utilizing telemedicine for improving heart failure readmission: can a realist approach bridge the translational divide? Clinical Medicine Insights Cardiology. 2019; 13: 1179546819861396.

[36] Paré G, Jaana M, Sicotte C. Systematic review of home telemonitoring for chronic diseases: the evidence base. Journal of the American Medical Informatics Association. 2007; 14: 269-277.

[37] Pandor A, Thokala P, Gomersall T, Baalbaki H, Stevens JW, Wang $\mathrm{J}$, et al. Home telemonitoring or structured telephone support programmes after recent discharge in patients with heart failure: systematic review and economic evaluation. Health Technology Assessment. 2013; 17: 1-207.

[38] Volterrani M, Sposato B. Remote monitoring and telemedicine. European Heart Journal Supplements. 2019; 21: M54-M56.

[39] Brahmbhatt DH, Cowie MR. Remote management of heart failure: an overview of telemonitoring technologies. Cardiac Failure Review. 2019; 5: 86-92.

[40] Gensini GF, Alderighi C, Rasoini R, Mazzanti M, Casolo G. Value of telemonitoring and telemedicine in heart failure management. Cardiac Failure Review. 2017; 3: 116-121.

[41] Zhu Y, Gu X, Xu C. Effectiveness of telemedicine systems for adults with heart failure: a meta-analysis of randomized controlled trials. Heart Failure Reviews. 2020; 25: 231-243.

[42] Dierckx R, Inglis SC, Clark RA, Prieto-Merino D, Cleland JGF. Telemedicine in heart failure: new insights from the Cochrane meta-analyses. European Journal of Heart Failure. 2017; 19: 304306.

[43] Allida S, Du H, Xu X, Prichard R, Chang S, Hickman LD, et al. mHealth education interventions in heart failure. Cochrane Database of Systematic Reviews. 2020; 7: CD011845.

[44] Son YJ, Lee Y, Lee HJ. Effectiveness of mobile phone-based interventions for improving health outcomes in patients with chronic heart failure: a systematic review and meta-analysis. International Journal of Environmental Research and Public Health. 2020; 17: 1749.

[45] Veenis JF, Brugts JJ. Remote monitoring of chronic heart failure patients: invasive versus non-invasive tools for optimising patient management. Netherlands Heart Journal. 2020; 28: 3-13.

[46] Angermann CE, Störk S, Gelbrich G, Faller H, Jahns R, Frantz S, et al. Mode of action and effects of standardized collaborative disease management on mortality and morbidity in patients with systolic heart failure the Interdisciplinary Network for Heart Failure (INH) study. Circulation: Heart Failure. 2012; 5: 25-35.

[47] Chaudhry SI, Mattera JA, Curtis JP, Spertus JA, Herrin J, Lin Z, et al. Telemonitoring in patients with heart failure. New England Journal of Medicine. 2010; 363: 2301-2309.

[48] Cleland JGF, Louis AA, Rigby AS, Janssens U, Balk AHMM. Noninvasive home telemonitoring for patients with heart failure at high risk of recurrent admission and death: the Trans-European Network-Home-Care Management System (TEN-HMS) study. ACC Current Journal Review. 2005; 45: 1654-1664.

[49] Zhang J, Goode KM, Cuddihy PE, Cleland JGF. Predicting hospitalization due to worsening heart failure using daily weight measurement: analysis of the Trans-European Network-HomeCare Management System (TEN-HMS) study. European Journal of Heart Failure. 2009; 11: 420-427.

[50] Koehler F, Winkler S, Schieber M, Sechtem U, Stangl K, Böhm $\mathrm{M}$, et al. Telemedical Interventional Monitoring in Heart Failure (TIM-HF), a randomized, controlled intervention trial investigating the impact of telemedicine on mortality in ambulatory patients with heart failure: study design. European Journal of Heart Failure. 2010; 12: 1354-1362.

[51] Koehler F, Winkler S, Schieber M, Sechtem U, Stangl K, Böhm M, et al. Impact of remote telemedical management on mortality and hospitalizations in ambulatory patients with chronic heart failure the telemedical interventional monitoring in heart failure study. Circulation. 2011; 123: 1873-1880. 
[52] Koehler F, Koehler K, Deckwart O, Prescher S, Wegscheider K, Kirwan BA, et al. Efficacy of telemedical interventional management in patients with heart failure (TIM-HF2): a randomised, controlled, parallel-group, unmasked trial. Lancet. 2018; 392: 1047-1057.

[53] Ong MK, Romano PS, Edgington S, Aronow HU, Auerbach AD, Black JT, et al. Effectiveness of remote patient monitoring after discharge of hospitalized patients with heart failure: the better effectiveness after transition - Heart Failure (BEAT-HF) randomized clinical trial. JAMA Internal Medicine. 2016; 176: 310-318.

[54] Krum H, Forbes A, Yallop J, Driscoll A, Croucher J, Chan B, et al. Telephone support to rural and remote patients with heart failure: the Chronic Heart Failure Assessment by Telephone (CHAT) study. Cardiovascular Therapeutics. 2013; 31: 230-237.

[55] Lyngå P, Persson H, Hägg-Martinell A, Hägglund E, Hagerman I, Langius-Eklöf A, et al. Weight monitoring in patients with severe heart failure (WISH). a randomized controlled trial. European Journal of Heart Failure. 2012; 14: 438-444.

[56] Virani SA, Sharma V, McCann M, Koehler J, Tsang B, Zieroth S. Prospective evaluation of integrated device diagnostics for heart failure management: results of the TRIAGE-HF study. ESC Heart Fail. 2018; 5: 809-817.

[57] Gattis WA, Hasselblad V, Whellan DJ, O'Connor CM. Reduction in heart failure events by the addition of a clinical pharmacist to the heart failure management team: results of the Pharmacist in Heart Failure Assessment Recommendation and Monitoring (PHARM) Study . Archives of Internal Medicine. 1999; 159: 1939.
[58] Cajita MI, Gleason KT, Han H. A systematic review of mHealthbased heart failure interventions. Journal of Cardiovascular Nursing. 2016; 31: E10-E22.

[59] Kaczorowski J, Chambers LW, Dolovich L, Paterson JM, Karwalajtys T, Gierman T, et al. Improving cardiovascular health at population level: 39 community cluster randomised trial of Cardiovascular Health Awareness Program (CHAP). British Medical Journal. 2011; 342: d442.

[60] Patja K, Absetz P, Auvinen A, Tokola K, Kytö J, Oksman E, et al. Health coaching by telephony to support self-care in chronic diseases: clinical outcomes from the TERVA randomized controlled trial. BMC Health Services Research. 2012; 12: 147.

[61] Härter M, Dirmaier J, Dwinger S, Kriston L, Herbarth L, Siegmund-Schultze E, et al. Effectiveness of Telephone-Based Health Coaching for Patients with Chronic Conditions: A Randomised Controlled Trial. PLoS ONE. 2016; 11: e0161269.

[62] Driscoll A, Currey J, Tonkin A, Krum H. Nurse-led titration of angiotensin converting enzyme inhibitors, beta-adrenergic blocking agents, and angiotensin receptor blockers for people with heart failure with reduced ejection fraction. Cochrane Database of Systematic Reviews. 2015; 2015: CD009889.

[63] Steckler AE, Bishu K, Wassif H, Sigurdsson G, Wagner J, Jaenicke C, et al. Telephone titration of heart failure medications. Journal of Cardiovascular Nursing. 2011; 26: 29-36.

[64] Alonso-Betanzos A, Bolón-Canedo V. Big-data analysis, cluster analysis, and machine-learning approaches. Advances in Experimental Medicine and Biology. 2018; 1065: 607-626. 